\title{
Research on Obstacle Avoidance of Fire-Fighting Robot Based On Fuzzy Control
}

\author{
Y.C. Lei, X.J. Zhu \\ School of Mechanical Eng ineering \\ Ning xia University \\ China
}

\author{
C.R. Tai \\ Shanghai Ingenious Automation Technology Co.Ltd. \\ China
}

\begin{abstract}
Fire-fighting robot can instead of humans in the event of a fire extinguishing task. An obstacle avoidance strategy based on fuzzy control is presented in this paper. The method doesn't have to determine the motion model of fire-fighting robot accurately. Robot can detect obstacles by using infrared sensor and detect the location of the fire with far infrared sensor. The environmental information which is detected by those sensors controls the robot movement and realizes the obstacle avoidance and navigation. Verified by the experiment, this method is used to control fire-fighting robot to detect the obstacles real-timely, to avoid obstacles effectively and to find a valid path in an unknown environment.
\end{abstract}

Keywords-fire-fighting robot; fuzzy control; obstacle avoidance

\section{INTRODUCTION}

Many scientific research institutions at home and abroad have begun to study fire-fighting robot, and it has been applied to the actual fire scene. Our country fire-fighting robots have been used from laboratory to practical application [1]. They will completely replace human fire-fighting task in a comple $\mathrm{x}$ environment and realize the intelligent, will own independent making decision ability and will avoid obstacles on the path in the working environment and find a safe path to the destination [2]. Intelligent robots detect environmental information depending on a variety of sensors and avoid obstacle based on the different strategies [3].

Fuzzy control is a kind of control method using fuzzy set theory, which does not need precise mathematical model, but by the person's experience and decision to the corresponding fuzzy logic reasoning. The fuzziness of language is used to describe the time-varying control process [4]. Fuzzy logic algorith m not only needs to construct the object motion model, but also the demand of the precision of data obtained from perception system is not high. So we can let the robot's action show more excellent uniformity, stability and no discontinuity, more suitably for real-time requirements under the condition of higher robot obstacle avoidance planning [5, 6, and 7]. In this paper, the robot realizes the obstacle avoidance by setting the fuzzy controller and verifies the feasibility of the autonomous obstacle avoidance through experiments.

\section{OVERALL STRUCTURE OF THE FIRE- FIGHTING ROBOT}

There are two driving wheels on the front of robot and a guide wheel on the behind of robot. Fire-fighting robot adopts differential drive way. The differential mode makes two independent movements synthesize into a sport. Two independently DC motors control the two driving wheels. The front of the robot is equipped with sensor system and the upper expansion bracket can be installed sensors. The installation of different sensors can satisfy the requirement of experiment. Liquid crystal display with a touch screen function and provides a good human-computer interaction interface, can display the sensor information. The overall structure and physical diagram are as shown in fig.1 and fig. 2 .

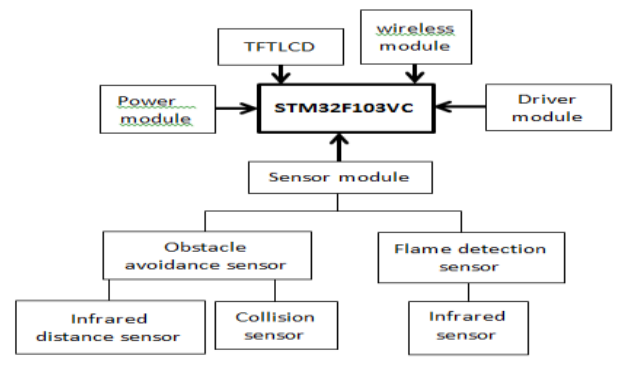

FIGURE I. OVERALL ST RUCT URE OFTHE ROBOT.

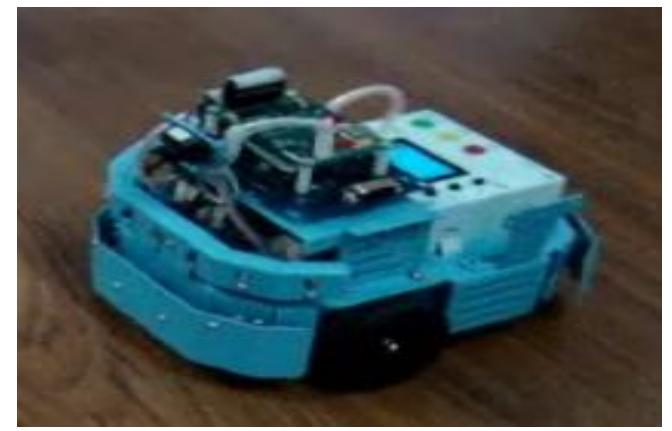

FIGURE II. THE PHYSICAL DIAGRAM OFTHE ROBOT.

\section{SENSOR S YSTEMS}

Sensors used to measure the distance and detect flame. We use the infrared sensor to detect obstacles. Infrared transmitting tube transmits infrared. Infrared receiving tube receives the infrared which reflected back after the obstacles. Infrared sensor of the robot can see ahead from $10 \mathrm{~cm}$ to $80 \mathrm{~cm}$.In addition, we also adopt collision sensors to implement emergency obstacle avoidance. Collision switch (normally open) and collision jo intly constitute the collision sensors, before and after the expansion in the robot. Collision ring is flexibly connected with the chassis. It occurs relative displacement after stressing, then triggers corresponding collision switch on the chassis. Collision generates a signal when switch collision switch is closed. The robot can achieve 
emergency obstacle avoidance after receiving the signal, which can avoid robots locked in dynamic obstacles or other sensors with an obstacle of the blind area.

Far infrared sensor can detect flame. They mainly are used to detect the front, left front and right front of the robot. The range of detection distance is from 0 to $1 \mathrm{~m}$. Far infrared sensors make the change from the external infrared light into current which make voltage produced on the resistance. The stronger the external infrared light, the smaller the value. According to the variation of the return value to determine the strength of the infrared light, we can identify different distance of heat source. Far infrared sensor detection angle is $60^{\circ}$. The distribution of sensors on the robot is shown in fig.3.

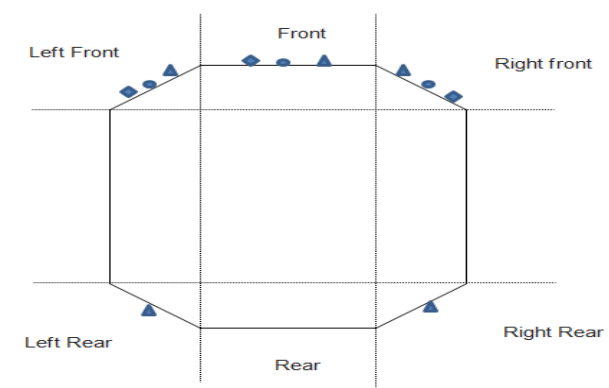

FIGURE III. $\triangle$ THE DIST RIBUTION OF ROBOT SENSORS. REPRESENT COLLISION SENSORS; REPRESENT INFRARED DISTANCE SENSORS; $\bigcirc$ REPRESENT INFRARED SENSORS.

\section{ANALYSIS ROBOT MOTION}

Control wheels of robot are driven by two DC motors. DC motor inputs shaft rotation motion to the gear box, and then the gearbox output shaft controls wheel rotation. The two wheels drive the whole movement of the robot. Mobile robot motion model is as shown in fig. 4 .

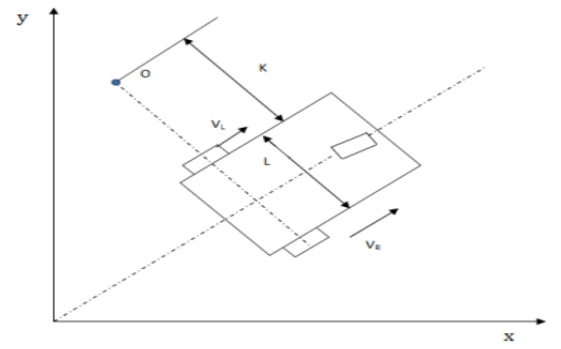

FIGURE IV. FIRE-FIGHTING ROBOT MOTION MODEL.

$\mathrm{K}$ represents center of rotation to the distance of the firefighting robot (radius of gyration); ${ }^{{ }_{L}}$ represents the speed of left wheel; $v_{R}$ represents the speed of the right wheel; 1 represents the distance of two driving wheels.

By the diagram, we can know turn radius are not equal. Angular velocity of left wheel can be written as follows:

$$
\omega_{L}=\frac{v_{L}}{K}
$$

Angular velocity of right wheel can be written as follows:

$$
\omega_{R}=\frac{v_{R}}{K+L}
$$

We can get the angular velocity of the robot:

$$
\omega=\omega_{L}=\omega_{R}=\frac{v_{R}-v_{L}}{L}
$$

The speed of the robot can be written as follows:

$$
\mathrm{v}=\omega(K+L / 2)=\frac{v_{R}+v_{L}}{2}
$$

When the speed of right wheel is equal to the speed of left wheel, the robot goes straight line. Under the condition of the real time expression:

$$
\begin{aligned}
\mathrm{v}(\mathrm{t}) & =\left(\nabla_{R}(\mathrm{t})+v_{L}(t)\right) / 2 \\
\omega(t) & =\left(v_{R}(t)-v_{L}(t)\right) / L
\end{aligned}
$$

We adjust the $v_{R}$ and $v_{L}$ to realize the movement of the robot.

The speed of DC motor:

$$
n=\frac{U_{\mathrm{a}}}{C_{\mathrm{E}} \phi}-\frac{R_{\mathrm{a}}}{C_{\mathrm{E}} C_{\mathrm{T}} \phi^{2}} T
$$

We can change the voltage $U_{a}$ to adjust speed. The relationship of robot rotational speed and torque is shown in figure 5 .

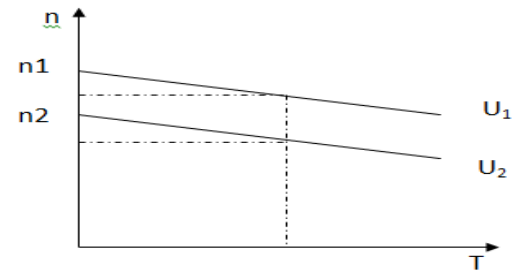

FIGURE V.THE RELATIONSHIP OF ROBOT.

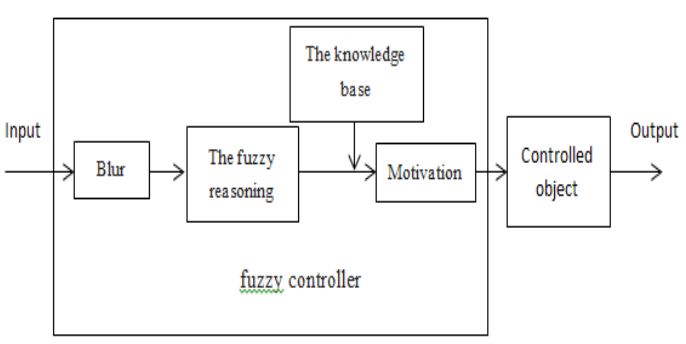

FIGURE VI. ST RUCTURE OF FUZZY CONT ROLLER ROTATIONAL SPEEDANDTORQUE.

When the load is constant (i.e. a certain torque) and we reduce the voltage $\left(U_{1}>U_{2}\right)$, the corresponding rotational speed is different $\left(n_{1}>n_{2}\right)$.so it can realize changing the speed of robot. We adopt PWM to adjust the voltage. Then the speed of robot will change.

\section{DESIGN A FUZZY OBSTACLE A VOIDANCE CONTROLLER}

In the process of the movement of the mobile robot, if the robot can avoid obstacles finding out a path to reach the target point safety or not is the key to realize the intelligent of mobile robot. How to choose a robot obstacle avoidance strategy to avoid obstacles is the key to find a safe path. 


\section{A. Introduction of Fuzzy Control}

Fuzzy control is a computer numerical control technology based on fuzzy set theory, fuzzy language variables and fuzzy logic reasoning. It is a essential kind of nonlinear control. There are a large number of practical application backgrounds. They belong to the category of the intelligent control. Fuzzy control becomes a very important branch in the automatic control field. The basic idea is to make a particular control strategy of controlled object or process summarized by the human experts into a series of "IF (condition) THEN (function) "statement. Then obtains corresponding amount of control collection through fuzzy inference and applied to controlled object or the environment. Fuzzy controller structure is as shown in fig. 6 .

\section{B. Fuzzy Control Rules}

The input of the fuzzy controller is the sensor distance information. The output is the turning angle of robot. Robot turning appropriate angle directly affect the real-time performance and stability of the robot obstacle avoidance. First blur input and output. The robot area is divided into three parts: the left front, front, right front. Domain of the theory is the distance of the infrared sensor. Obstacle distance in front is QD. Left obstacle distance in front is LD. front obstacle distance is RD. The information about the distance as an input is different between left front and right front (ED).So the inputs of the fuzzy controller are $\mathrm{QD}$ and $\mathrm{ED}$ and $\mathrm{T}$ is as output.

\section{1) The fuzzy treatment}

After blurring, the QD changes to $\{\mathrm{Y}, \mathrm{Z}, \mathrm{J}$, and L\}.They respectively represent the distance from robot to barriers: far, near, close and without obstacles. ED blur changes to $\{R Y, R Z$, RJ, L LY, LZ, LJ $\}$. They respectively represent the distance which indicate far on the right side, near on the right side, close on the right side no obstacles, far on the left side, near on the left side and close on the left side. T blurs changes to \{TL4 TL3, TL2, TL1, TZ, TR1, TR2 TR3, and R4\}.They represent the angle of robot under different obstacle distance. The me mbership function of input and output choose symmetrical triangle which is as shown in fig.7, fig.8 and fig.9.

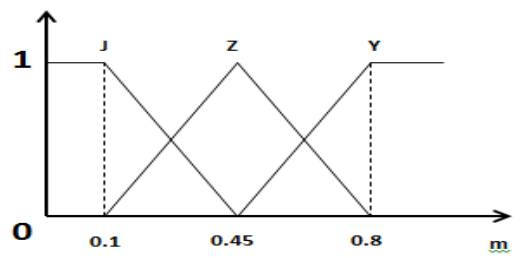

FIGURE VII. MEMBERSHIP FUNCTION OF QD.

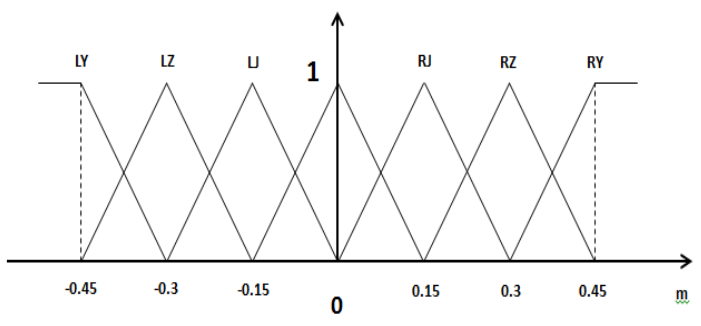

FIGURE VIII. MEMBERSHIP FUNCTION OF ED.

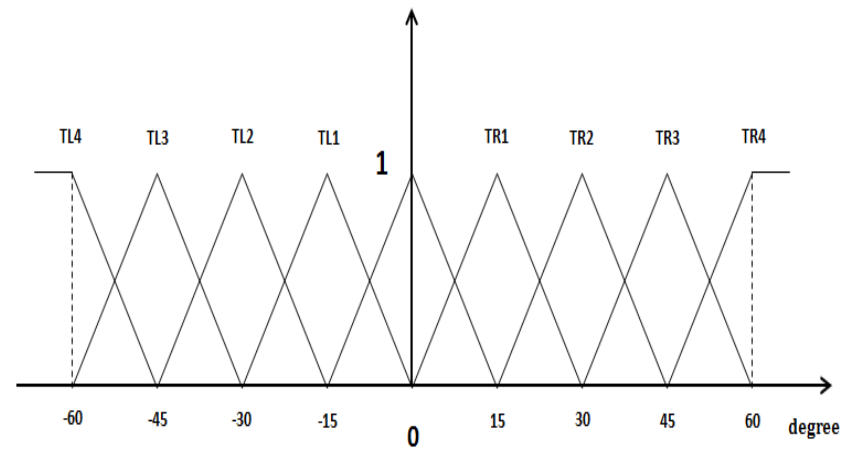

FIGURE IX. THE MEMBERSHIP FUNCTIONS OF OUTPUT VARIABLES $\mathrm{T}$.

\section{2) Design of rule base and fuzzy inference}

The core of the fuzzy control is to establish the appropriate fuzzy rules. The QD and ED is respectively as input variables in the design of the fuzzy subset containing four and seven rules, and $\mathrm{T}$ is as the output variable. So the fuzzy rules library contained 28 corresponding rules in article. But when the QD is equal to zero, robots do not do any obstacle avoidance behavior. So the simplified fuzzy rule base with 22 rules is as shown in table 1.

TABLE I. THE FUZZY RULE BASE.

\begin{tabular}{|c|c|c|c|c|c|c|c|}
\hline number & \multicolumn{2}{|c|}{ Input } & \multirow{2}{*}{ Output } & number & \multicolumn{2}{|c|}{ Input } & \multirow{2}{*}{ Output } \\
\cline { 2 - 3 } & QD & ED & & & QD & ED & \\
\hline 1 & Y & RY & TR1 & 12 & Z & LY & TL2 \\
\hline 2 & Y & RZ & TR2 & 13 & Z & LZ & TL2 \\
\hline 3 & Y & RJ & TR2 & 14 & Z & LJ & TL3 \\
\hline 4 & Y & L & TR1 & 15 & J & RY & TR3 \\
\hline 5 & Y & LY & TL1 & 16 & J & RZ & TR3 \\
\hline 6 & Y & LZ & TL2 & 17 & J & RJ & TR4 \\
\hline 7 & Y & LJ & TL2 & 18 & J & L & TR3 \\
\hline 8 & Z & RY & TR2 & 19 & J & LY & TL3 \\
\hline 9 & Z & RZ & TR2 & 20 & J & LZ & TL3 \\
\hline 10 & Z & RJ & TR3 & 21 & J & LJ & TL4 \\
\hline 11 & Z & L & TR2 & 22 & L & & TZ \\
\hline 3$)$
\end{tabular}

The output of the control signal which launched by fuzzy library is not clear digital quantity. Well we want to have a precise value to control the robot. The most common methods in fuzzy control include maximum membership degree method, the center of area method and area divided method, etc. Because the area average method is reasonable and has a simple calculation. This paper adopts the area average method to blur.

\section{Obstacle Avoidance Program Design}

The main control chip judges the obstacles by reading sensor signal. The clear numerical value is obtained by fuzzy controller which drive robot turns a certain angle to realize the effectively obstacle avoidance for the robot. Obstacle avoidance of the main program flow is as shown in fig. 10 . 


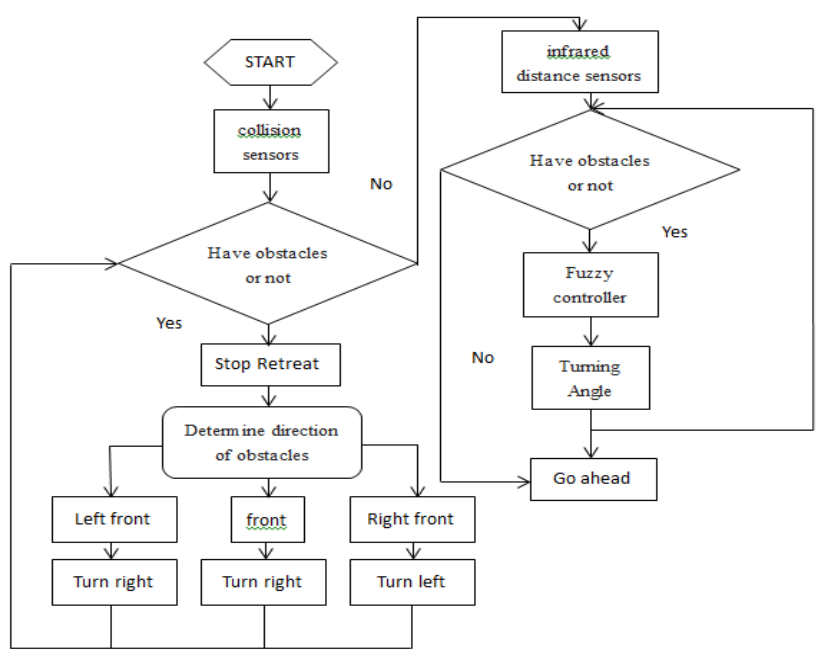

FIGURE X. OBSTACLE AVOIDANCE OFT HE MAIN PROGRAM.

\section{THE EXPERIMENT AND CONCLUSION}

The obstacle avoidance test is performed in laboratory environment. At first, place a few regular square barriers. The results show that the fire-fighting robots effectively avoided obstacles to seek the fire. And then we increase the number of obstacles. We find that when obstacles appear "lane" which the robot cannot pass, robots swing and be locked. In the program we join safety judged into robot by the width of the "lane" which the robot cannot pass. Then the robot avoids the "lane" looking for other paths. But it takes more time to find the fire.

Fuzzy control is verified by experiment that can effectively avoid obstacles. But in the more complex or dynamic obstacles can also have a problem. In the later work, putting some algorithms together will be ab le to reduce the limits of an algorith $\mathrm{m}$. So we can fusion other algorith $\mathrm{ms}$ on the basis of fuzzy control. It can save more time to avoid obstacles and to look for a safe path.

\section{REFERENCES}

[1] Jian-guo Liu, Yu-hong Du. Design application of Fire-fighting robot. Mechanical and electrical engineering, 2009.

[2] Xian-kong Ding.Introduce the application of fire-fighting robot in fire fire Shanghai fire research institute, 2004, 8.

[3] Guang-wu Hu, Sheng Zhao, Qing-peng Ren Etc.Design of Intelligent Fire-fighting Robot Based on STM32 Control. Automation Application, 2014,7.

[4] Mohammad Mehdi Fateh, Sara Fateh. A Precise Robust Fuzzy Control of Robots Using Voltage Control Strategy. International Journal of Automation and Computing, 2013, Vol.10, pp.64-72.

[5] Wang Chong. Simulation Research on Car Intelligent Barrier Avoidance Method. Computer Simulation,2014,6.

[6] Hua-zhi Chen, Cun-xi Xie. FuzZy Control of Obstacle Avoidance for Mobile Robot. Machine Tool \& Hydraulics,2004,11.

[7] Yu-chao Lu, Ke-cun Jin, Yu-tian Liu.The Study of Humanoid Robot Gait Stability Based on Fuzzy Control Method. Artificial Intelligence and Robotics Research, 2014, Vol.03 (01), pp.11-17. 